\title{
Projeto Bandeira Científica: descrição e análise crítica de uma experiência pioneira de extensão universitária
}

\author{
"Bandeira Científica" (Scientific Expedition): description and critical analysis \\ of a pioneer extension program
}

\author{
Gabriela Queiroz do Amaral', Paulo Henrique dos Santos Mota ${ }^{2}$
}

\begin{abstract}
Amaral GQ, Mota PHS. Projeto Bandeira Científica: descrição e análise crítica de uma experiência pioneira de extensão universitária / "Bandeira Científica" (Scientific Expedition): description and critical analysis of a pioneer extension program. Rev Med (São Paulo). 2017 jan.-mar.;96(1):21-6.
\end{abstract}

RESUMO: O Projeto Bandeira Científica é um dos mais antigos Projetos de Extensão da Faculdade de Medicina da Universidade de São Paulo (FMUSP), colaborando desde sua criação para o fortalecimento da Educação Médica. Originalmente fundado em 1957, o Projeto passou desde então por várias transformações, reconstruções e fases de maturação. Atualmente tem como foco atuar em áreas de vulnerabilidade em saúde, desenvolvendo não só atividades de assistência, como também de prevenção e promoção à saúde. Ao longo do tempo, foi se tornando cada vez mais multidisciplinar, contando com a participação de diversos cursos da USP ligados direta ou indiretamente à saúde, além de estabelecer parcerias com universidades locais situadas próximos as cidades visitadas a cada ano. Busca ser um espaço transformador, onde estabelecer o diálogo com gestores e prestadores de serviços, elaborar propostas de intervenção e ir ao encontro de uma população com suas peculiaridades, possam ser fatores que desenvolvam a um processo de reflexão crítica nos alunos sobre as condicionantes histórico-estruturais da saúde no Brasil.

Descritores: Promoção da saúde/recursos humanos; Estudantes de ciências da saúde; Estudos interdisciplinares; Assistência; Relações interprofissionais; Ciências da saúde/educação; Expedições/normas; Relações comunidade-instituição.

\begin{abstract}
Bandeira Científica" (Scientific Expedition) is one of the oldest Extension Projects from University of São Paulo Medical School (FMUSP), working since its inception to embrace medical education. The project has gone through several transformations, reconstructions and stages of maturation since its foundation in 1957. Currently focusing at providing health care in vulnerable cities of Brazil, achieving not only assistance activities, but also health prevention and promotion. Over time, it became increasingly multidisciplinary, with the communion of several courses from University of São Paulo directly or indirectly related to health. The project also establishes partnerships with universities located near the cities visited each year. Scientific Expedition aims to be a transformative program, establishing dialogue with service providers to draw up proposals for intervention and meeting a population reality with its peculiarities, enabling the development of critical reflection by the students about the health historical-conditioning factors in Brazil.
\end{abstract}

Keywords: Health promotion/manpower; Education, medical, undergraduate; Interdisciplinary studies; Assistance; Interprofessional relations; Health sciences/education; Expeditions/standards; Community-institutional relations.

1.Acadêmica da Faculdade de Medicina da Universidade de São Paulo. E-mail: gabriela.amaral@fm.usp.br.

2.Mestre em Ciências, Preceptor de Graduação do Departamento de Fisioterapia, Fonoaudiologia e Terapia Ocupacional da Faculdade de Medicina da Universidade de São Paulo.E-mail: paulo.mota@fm.usp.br.

Autor responsável : Gabriela Queiroz do Amaral. Rua Oscar Freire, 1753, Apart. 41B. CEP: 05409011 - São Paulo, SP. E-mail: gabriela. amaral@fm.usp.br. 


\section{INTRODUÇÃO}

Projeto Bandeira Científica (BC) é um dos
mais antigos e impactantes Projetos de Extensão da Faculdade de Medicina da Universidade de São Paulo (FMUSP), colaborando desde sua criação para o fortalecimento da Educação Médica. Tem como missão contribuir para a formação social, acadêmica e profissional dos estudantes da Universidade de São Paulo, por meio de ações que visam melhorar as condições de saúde de localidades vulneráveis do Brasil.

Dessa forma, se propõe a ser um projeto de extensão universitária modelo na abordagem global e interdisciplinar da problemática em saúde das comunidades vulneráveis com impacto no desenvolvimento social e econômico, garantindo a sustentabilidade e aliando a isto a capacitação técnica, social e humanística de profissionais em formação.

No que tange o conceito de saúde atualmente admitido pelo Projeto, adota-se a definição da Organização Mundial da Saúde (OMS), em que saúde é entendido como estado de completo bem estar físico, mental e social e não apenas a ausência de doença ${ }^{1}$.

Atua prioritariamente através de ações voltados para práticas em saúde. Estabelece relações com a gestão do município atendido e busca criar parcerias com os diferentes atores sociais da cidade assistida para desenvolver ações onde são levadas em conta principalmente as demandas locais de saúde. Isso não significa que demandas de outros setores não diretamente relacionadas à saúde não serão atendidas; entretanto, deve-se primeiramente garantir que os objetivos primordiais do Bandeira Científica sejam cumpridos, para depois realizar ações adicionais.

Entre o elenco de atividades propostos pelo Projeto estão: "ações preventivas individuais": aqui estão contempladas atividades educativas, de conscientização e demais ações de prevenção que apresentem impacto sobre o indivíduo, mesmo que realizadas coletivamente (ex: grupos específicos de pessoas, como gestantes); “ações estruturais de impacto direto": engloba ações maiores que possam impactar positivamente na saúde de forma direta (ex: construção de fossas, fluoretação da água do município); "ações terapêuticas direcionadas": contempla todos os atendimentos médicos em ação conjunta com outras áreas da saúde, visitas domiciliares e demais atividades que envolvam o tratamento clínico de indivíduos ou de grupos.

\section{Contextualização histórica}

Analisando-se o histórico do Bandeira Científica, pode-se dividir a trajetória do projeto em quatro fases fundamentais, que traduzem sua evolução ao longo do tempo, evidenciando a constante renovação e reconstrução pelas quais passou. Na Figura 1 são mostradas os municípios visitados desde 1998.
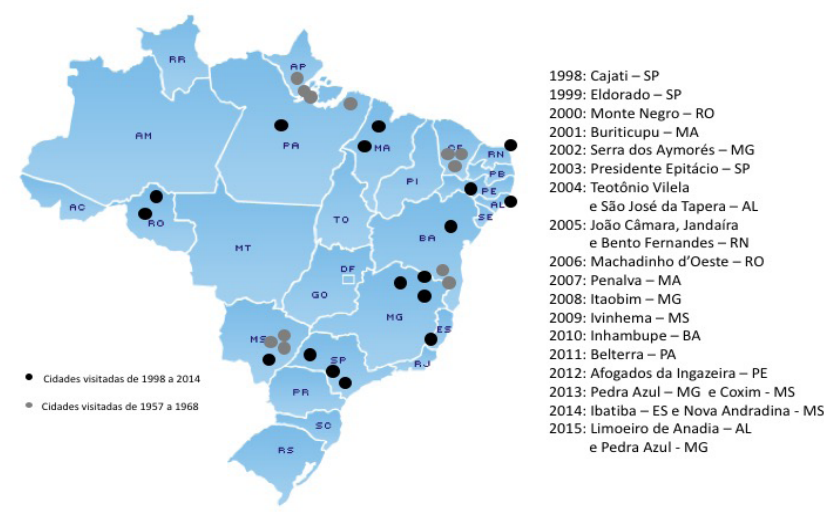

Figura 1. Municípios visitados pelo Projeto Bandeira Científica

\section{Fase 1: Da criação à interrupção abrupta (1957-1969)}

O Projeto Bandeira Científica foi criada em 1957 por iniciativa de Alexandre F. M. Lourenço, da $44^{\mathrm{a}}$ Turma de Medicina da FMUSP, que, com o apoio de outros alunos da Casa, idealizou uma expedição ao Pantanal do Mato Grosso para a realização de atividades de pesquisa, sob a coordenação do professor Luis Rey. Esta primeira expedição durou cerca de um mês, cobrindo quatro cidades da região para coleta de informações de saúde. A adesão foi tamanha que, em 1959, foi realizada uma nova expedição, desta vez para Pernambuco. Seguiram-se mais nove expedições, dirigindo-se a municípios do Ceará, Pará, Amapá, Bahia e Rio Grande do Sul. Em 1969, as atividades do Projeto foram interrompidas devido à realidade políticosocial da época - incluindo a aposentadoria compulsória dos professores Luís Rey e Luiz Hildebrando Pereira da Silva, grandes entusiastas e apoiadores do projeto $^{2}$.

\section{Fase 2: A refundação (1998-2001)}

Após uma latência de quase trinta anos, em 1997, o acadêmico Rafael Bernardon Ribeiro, da $85^{\text {a }}$ Turma de Medicina da FMUSP descobriu o Projeto consultando arquivos da Faculdade e mobilizou-se para reativá-lo, com o apoio e coordenação do professor Paulo Hilário Nascimento Saldiva, do Departamento de Patologia. Além das atividades fundamentais de ensino e de pesquisa herdadas do conceito inicial da Bandeira -, a partir de 1999 foi introduzida a vertente assistencial, materializada no atendimento básico em nível primário à população local, visando a elaboração de diagnóstico populacional de saúde para os municípios visitados. Isto representou um grande avanço na contribuição social do projeto para a comunidade visitada, além de uma experiência adicional e inédita para os alunos da FMUSP. Assim, em 2000, O Projeto Bandeira Científica tornou-se projeto de extensão universitária da USP .

A partir do mesmo ano, sob a coordenação do professor Carlos Corbett, do Departamento de Patologia, 
o projeto passou a atuar fora do estado de São Paulo. No sentido de garantir a sustentabilidade e continuidade das ações, foram feitas parcerias com universidades locais. Assim, desenvolveram-se as expedições de Monte Negro (RO) em 2000 e Buriticupu (MA) em 2001².

\section{Fase 3: Maturação e multidisciplinaridade (2002-2007)}

Durante os três anos seguintes (expedições em 2002, 2003 e 2004), o Projeto tomou corpo, amadurecendo a sua forma de operação e desenvolvimento de atividades. O crescimento dos então denominados programas de Saúde da Família passou a garantir maior acesso da população à atenção básica e a gerar maior demanda por atendimentos especializados. A Bandeira se adaptou a isto, ampliando o leque de especialidades médicas envolvidas no projeto e incluindo também, pela primeira vez, uma equipe de alunos e professores do curso de Fisioterapia da USP, o que se tornaria o embrião de uma nova e produtiva alteração conceitual do projeto.

A análise periódica dos dados coletados nas expedições mostrou que a atuação da equipe médica tinha efeitos importantes, mas que também tinha algumas limitações, especialmente resultantes do conceito mais restrito de saúde adotado pelo Bandeira Científica até então. Nesta nova fase, novamente sob a supervisão do professor Paulo Saldiva e do doutor Luiz Fernando Silva, foi oficialmente adotada a definição de saúde da OMS ("completo estado de bem estar físico, mental e social"). Isto resultou na progressiva incorporação de outras áreas do conhecimento, iniciando-se com a Nutrição na expedição de 2005; culminando com a inclusão da Odontologia, Psicologia, Agronomia e das Engenharias Civil e Ambiental na expedição de 2006; e a inclusão do Jornalismo e do Audiovisual em 2007².

\section{Fase 4: A interdisciplinaridade e a sustentabilidade} (2008-2012)

A inclusão de novas áreas do conhecimento abriu também novos horizontes para o projeto. No início, elas atuavam no mesmo local, mas de modo específico, formando um projeto com múltiplas atividades não finamente alinhadas entre si. Com o passar do tempo, os próprios alunos de cada área passaram a conhecer melhor as atividades das outras, o que permitiu o avanço no sentido de se desenvolverem ações interdisciplinares nos diferentes pontos de atendimento.

Assim foram as expedições de 2008 a 2011. Para potencializar esta interação, a partir de reuniões interdisciplinares, foram elaborados projetos conjuntos, como interação Engenharia e Fisioterapia para construção de cadeiras de rodas; Odontologia, Fonoaudiologia e Psicologia na atenção à saúde bucal; Odontologia e Fisioterapia para a ergonomia dos participantes;
Agronomia, Medicina e Fisioterapia para atuação nas comunidades rurais afastadas; visitas domiciliares com até oito áreas envolvidas, dependendo das necessidades dos casos, entre outros. Em 2012, a Terapia Ocupacional foi integrada à Bandeira Científica.

Além disso, buscou-se garantir a sustentabilidade e continuidade das atividades realizadas através do reforço na elaboração de projetos estruturais para os municípios nas áreas de saúde e saneamento; formação e capacitação de multiplicadores e profissionais locais de saúde; estímulo ao desenvolvimento de projetos de extensão semelhantes pelas universidades parceiras; uso de ferramentas de comunicação à distância para acompanhamento de indicadores e apoio às cidades ${ }^{2}$.

\section{Fase 5: Transição e Continuidade (2013- ?)}

A atual fase do Bandeira Científica é caracterizada por três aspectos: melhorar as formas de gestão do projeto; estruturar a relação com os municípios atendidos e incremento das atividades realizadas.

Neste período o Projeto aconteceu sob supervisão do professor Luiz Fernando Silva e do Doutor Paulo Mota, que, juntamente a profissionais que no passado atuaram como alunos no projeto, formam uma equipe que visa dar maior apoio as ações realizadas pelos alunos em todas as etapas dos projetos anuais, desde sua concepção, gestão e execução.

No aspecto das relações com os municípios, há o entendimento de que o Bandeira Científica torna-se um ponto de ação da rede de atenção à saúde do município e dessa forma deve dialogar com o sistema local, buscando uma integração que, em última instância, amplie o cuidado do paciente atendido.

Por fim, neste período é também criada uma segunda expedição anual do Projeto, mas desta vez com foco cirúrgico, onde são realizados procedimentos de ginecologia, cirurgia geral, anestesiologia, radiologia e patologia.

\section{Edições e municípios visitados}

Após a sua reativação em 1998, o Projeto Bandeira Científica esteve presente nos municípios apresentados na Figura 1.

\section{RESULTADOS}

Ao longo dos últimos 16 anos, com as transformações apresentadas, diversas são as formas de se mensurar resultados para o Projeto. No presente trabalho será enfocado os números gerais, destacando-se ações que levaram a alterações sistêmicas na construção do Bandeira Científica. 


\section{Número de participantes}

Conta atualmente com alunos de 13 cursos de graduação da Universidade de São Paulo, pertencentes a 7 unidades, Faculdade de Medicina, Faculdade de Saúde Pública, Instituto de Psicologia, Faculdade de Odontologia, Escola Politécnica, Faculdade de Economia, Administração e Atuária e Faculdade de Ciências Farmacêuticas.
Já participaram das atividades de campo do Bandeira Científica 2802 pessoas, sendo 2076 alunos e 585 profissionais. No Gráfico 1 pode ser visto a evolução do número de participantes havendo destaque para o expressivo aumento em 2007 devido a incorporação de diversos cursos novos e queda ocorrida em 2013 que se manteve no ano seguinte em virtude das alterações de gestão enfrentadas pelo Projeto.

Gráfico 1. Número de alunos e profissionais envolvidos nas expedições do Projeto Bandeira Científica. São Paulo (2015)

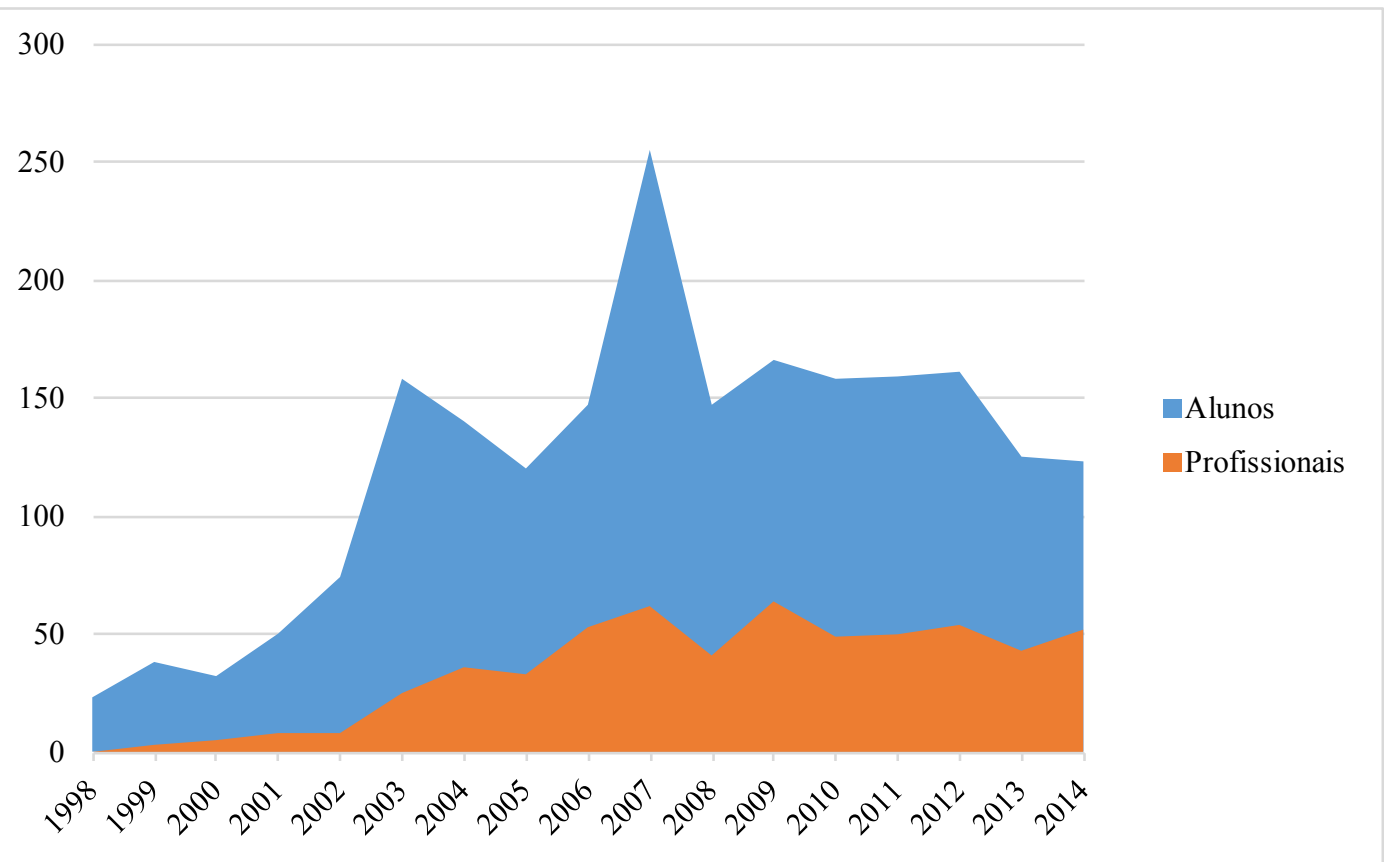

É essencial destacar ainda a forte presença de graduandos e profissionais de universidades próximas aos municípios onde ocorrem as atividades. Já participaram 451 alunos e 51 profissionais, com destaque para a expedição de 2007 que contou com 148 participantes. No Gráfico 2 são apresentadas as universidades parceiras e a porcentagem da participação de cada equipe ao longo dos anos.

Gráfico 2. Porcentagem de participantes de universidades parceiras. São Paulo (2015)

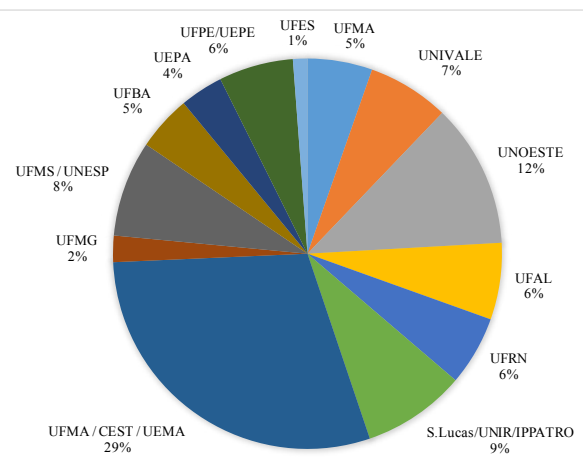

\section{Ação comunitária na experiência multiprofissional}

Ao longo de sua recente existência já foram realizadas 16 expedições multiprofissionais e 3 expedições cirúrgicas. Foram visitados 22 municípios de 11 unidades da federação. O Projeto já esteve em todas as macroregiões brasileiras com exceção da macroregião Sul.

As transformações vividas pelo projeto Bandeira Científica ao longo de sua trajetória histórica vieram em grande parte devido ao contato com as necessidades locais das comunidades acompanhadas, mas também ocorreram por novas ambições e demandas de aprendizado dos alunos.

A realização de atendimentos em saúde torna-se portanto um caminho que abre um diálogo novo com a comunidade. Entende-se que existe a necessidade de discutir novas formas de manutenção da saúde tanto a nível dos usuários dos serviços, quanto no que tange as gestões municipais de saúde. Criam-se então atividades de cunho coletivo, onde são criados espaços de discussão e tomada de decisão entre os alunos, os profissionais e a população da cidade assistida.

Ao mesmo tempo percebe-se a necessidade de estabelecer uma relação mais efetiva com os profissionais 
locais criando-se mecanismos de contra-referência atendimentos realizados e estabelecendo-se as ações a serem executadas através de um contato mais próximo com o sistema local.

No Gráfico 3 pode ser visualizada a produção de atividades realizadas desde 1999 pelo projeto.

Gráfico3. Número de atividades realizadas por ano no Projeto Bandeira Científica. São Paulo (2015)

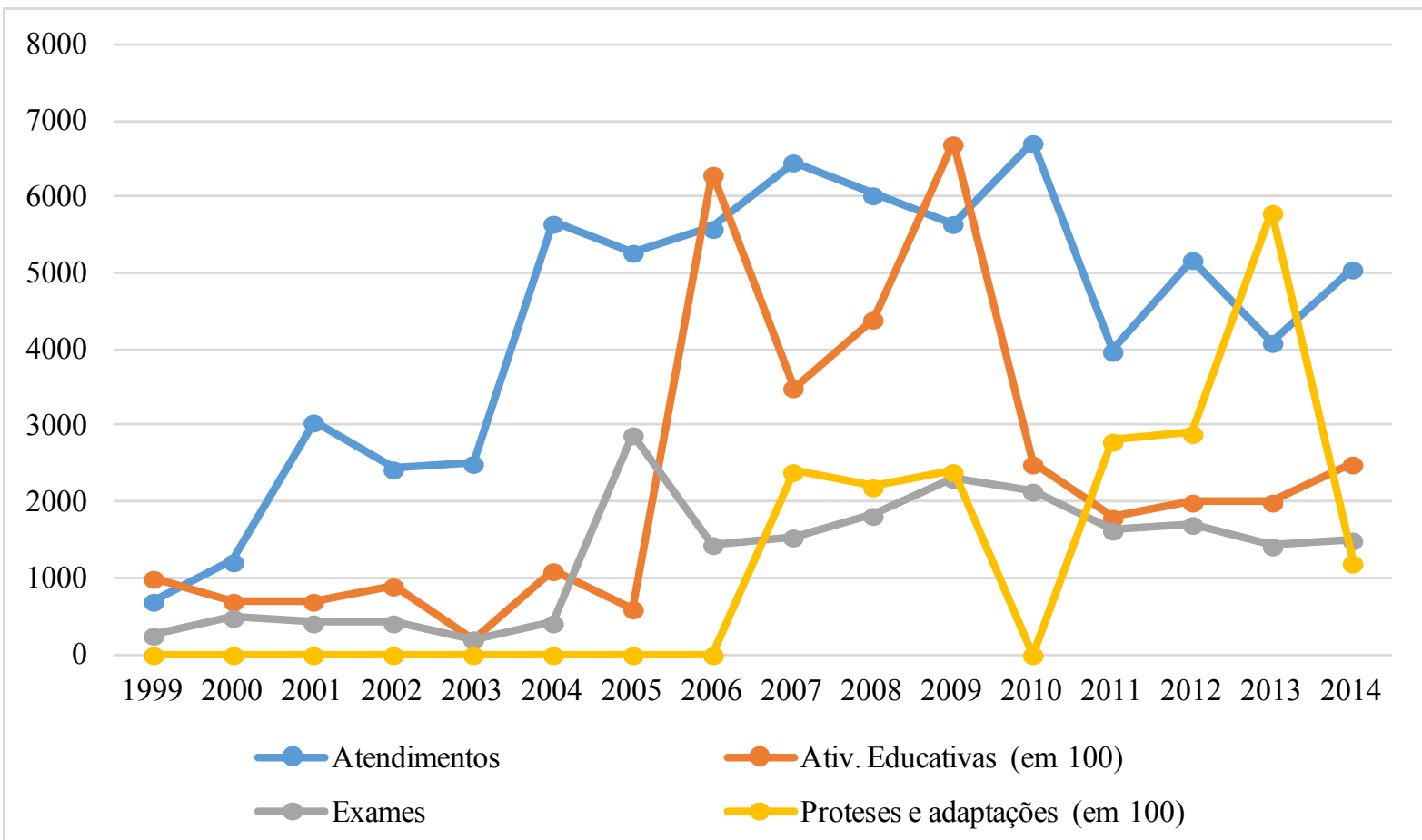

Nota-se uma substancial diminuição dos atendimentos e atividades coletivas/educativas realizados durante as ações. A partir de 2011, com um segundo momento de expansão dos cursos participantes, começou a ser preconizado um menor número de atividades, com o enfoque na qualidade das mesmas e na sua possibilidade de atingir maior número de pessoas através do aumento das ações construídas de forma multidisciplinar.

Anualmente ainda, são produzidos relatórios detalhados dos inquéritos epidemiológicos e sociais, bem como das diferentes atividades assistenciais realizadas. Todos os dados são analisados sob o prisma científico, buscando sempre a elaboração de perguntas e a busca de conclusões relevantes para a comunidade local.

A tabulação dos dados epidemiológicos e dos atendimentos e diagnósticos realizados busca traçar informações e levantar propostas que possam ser relevantes para os gestores refletirem e possivelmente implementarem medidas visando melhor qualidade do sistema de saúde local. Para os usuários são trazidas informações que podem contribuir para a tomada de decisão sobre sua própria saúde e sobre as condições gerais do município.

\section{Análise crítica}

A evolução apresentada pelo Projeto Bandeira Científica nos últimos anos ilustra um esforço que vai além da realização de atividades de puramente assistenciais. Estes enfoques têm sido anualmente expandidos e melhorados através de reuniões anuais de apresentação de dados e discussão de estratégias e resultados; da ampliação da interdisciplinaridade do projeto, e da elaboração de diferentes estratégias de continuidade ${ }^{2}$.

Dentro dessa perspectiva, torna-se evidente a necessidade de integração concreta do tripé básico da Universidade - ensino, pesquisa e extensão. Sem essa integração, dificilmente se poderá pensar que ela terá condições de preparar profissionais com a qualificação que o país exige. Não basta, entretanto, que ensino, pesquisa e extensão universitária se integrem "intramuros", tornase necessário que seja estabelecido também um contato estreito com a comunidade ${ }^{3}$.

A Extensão é, portanto, assumida como "um processo educativo, cultural e científico que articula o ensino e a pesquisa de forma indissociável e viabiliza a relação transformadora entre universidade e sociedade" ${ }^{4,5}$.

Para Ângelo ${ }^{6}$ a busca por novos caminhos para desenvolver a formação de novos profissionais passa pelo conceito de aprendizagem ativa, necessária para o desenvolvimento do pensamento crítico e de uma educação integral, em contraposição ao simples treinamento técnico.

Ao contrário de dados frios apresentados em salas de aula, da realidade de indicadores de saúde dos livros e artigos e da teoria da organização do Sistema Único de 
Saúde no Brasil, no Projeto Bandeira Científica o aluno é colocado no âmago da situação e, naquele período, passa a fazer parte dela. Neste processo ele passa a conviver lado a lado com as condições reais de vida e de assistência à saúde em nosso país e se depara com o desafio de trabalhar em situações com escassos recursos complementares para auxiliar os diagnósticos e exercer o manejo adequado dos casos clínicos, conhecendo então a realidade do atendimento em saúde vigente na maior parte dos municípios brasileiros.

Além disso, através de ações comunitárias realizadas, o aluno pode começar a perceber que há diferença entre enxergar exclusivamente a saúde individual do paciente e a saúde de uma população. Esta organizada a partir de um sistema ações e planejamento que correspondem ao Sistema Único de Saúde (SUS), que tem como princípios a universalidade, a equidade, integralidade a regionalização e hierarquização.

Para que tais premissas sejam cumpridas, ações como o Projeto Bandeira Científica são muito interessantes, pois nele ocorre uma importante integração: as novas perspectivas e novos saberes se fazem acompanhar de uma educação capaz de oferecer à sociedade profissionais cuja identidade seja carregada de elevada competência

\section{REFERÊNCIAS}

1. World Health Organization (WHO). Definition of health [cited 2015 Aug 25]. Available from: https://apps.who.int/aboutwho/ en/definition.html.

2. Silva LFF. Projeto Bandeira Científica: história, estratégias e resultados. Rev Med (São Paulo). 2012;91(1):36-43. doi: http:// dx.doi.org/10.11606/issn.1679-9836.v91i1p36-43.

3. Pereira MT. Extensão universitária na ESALQ-USP: o caso dos agricultores de São Pedro-SP. Estud Av (São Paulo). 2001;15(43):281-8. http://dx.doi.org/10.1590/S010340142001000300021 .

4. Garrafa V, organizador. Extensão: a universidade construindo técnico/científica e ética, mas, sobretudo profissionais comprometidos com a transformação das condições de vida e saúde da população ${ }^{7}$.

Existe uma proposta ainda em discussão de retorno a um município atendido há cerca de 5 anos pelo projeto para comparação de realidades e desenvolvimento de pesquisas qualitativas do papel da Bandeira ${ }^{2}$.

Os desafios do Projeto Bandeira Científica ainda são diversos e de grande dificuldade de concretização. Potencializar ações interdisciplinares, e principalmente organiza-las de forma a buscar maior coesão com o município assistido é provavelmente o objetivo mais desafiador no momento. Busca-se ainda dialogar, entender e se adaptar as novas realidades de saúde encontradas no Brasil, em decorrência das profundas mudanças pelo qual passou o país desde a refundação do Projeto.

Por fim, o Projeto Bandeira Científica pretende ser um espaço transformador, onde estabelecer o diálogo com gestores e prestadores de serviços, elaborar propostas de intervenção e ir ao encontro de uma população com suas peculiaridades possam ser fatores que desenvolvam um processo de reflexão crítica no alunos sobre as condicionantes histórico-estruturais da saúde no Brasil.

saber e cidadania: relatório de atividades, 1987/1988. Brasília: Ed. UNB; 1989.

5. Hunger D, Rossi F, Pereira JM, NOzaki JM. O dilema extensão universitária. Educ Rev (Belo Horizonte). 2014;30(3):335-54. Disponível em: http://scielo.br/pdf/edur/v30n3/edur640.pdf.

6. Angelo M, Educação em enfermagem: a busca da autonomia. Rev Esc Enferm USP. 1994;28(1):11-40. doi: http://dx.doi. org/10.1590/0080-6234199402800100011.

7. Santos AM, et al. Enfermagem em saúde pública: uma proposta articulando ensino, pesquisa, estágio e extensão. Rev Bras Enferm. 2000;53(spe):159-64. doi: http://dx.doi.org/10.1590/ S0034-71672000000700028. 